\title{
Data Classification and Personal Care Management System by Machine Learning Approach
}

\author{
Dr. G. Pradeepini ${ }^{1}$, G. Pradeepa $^{2}$, B. Tejanagasri ${ }^{3}$, Sri Harsha Gorrepati ${ }^{4}$ \\ ${ }^{1,2,3,4}$ CSE, K L E F, Vaddeswaram, India \\ *Corresponding author E-mail: pradeepini cse@kluniversity.in
}

\begin{abstract}
The ace system devices are expected as a key half inside the change of welfare as to such an extent as consistent discerning of patients treatment and preservation of E-pharmaceutical structure. The basic test that patients went up against is that the truth of problem in achieving specialist authorities. This paper proposes Associate in nursing keen structure which will give self-care what is a considerable measure of, checking system which will reproduce the patient in sight of his/her disorder. The methodology are at regardless of reason a patient sends his insight concerning his biopsy and entirely unexpected tests, the system can choose regardless of whether the condition is fundamental or not. In unimportant condition, the sharp system can give the recommendations of which psychological disorder he/she is facing. The structure used can revive information methodically with understanding and learning algorithms. A machine-learning estimation was directed to play out the gathering procedure.
\end{abstract}

Keywords:Psychological disorders; Machine Learning Algorithm; Nä̈ve Bayes; PTSD; ASD; Health-care.

\section{Introduction}

The basic idea is to create an application that can predict whether the patient is having a particular psychological disorder or not. For this as being a subject of extensive research, we are not provided any dataset of patients about the stages which are involved in $\mathrm{him} / \mathrm{her}$ being victim to a psychological disorder. Hence, we had to create the dataset where we involved two cases whether the patient is having PTSD or ASD. The program model of Naïve Bayes that we have designed using R determines how much probability the patient has to acquire PTSD or ASD based on the dataset that we have created of a particular patient. To come up with this idea we have taken the knowledge we acquired by using few honored research papers of the past particularly papers that dealt with SCD (Sickle Cell Disease). The improvement in correspondence and developments and their use in restorative stage have adequately altered the way of life in association with upgrading human administrations workplaces and results. it's in this way essential for patients United Nations office aptitude the tired impacts of RBC contamination (SCD) to be completely investigated at beginning sum determined treatment are regularly associated rapidly. The expanding assortment of DS patients has altered the treatment procedures from recuperating office mind toward out of mending office mind that wards all on data advancement (IT). There region unit various made countries presented Electronic energetic (E-prosperity) programs as to such an extent as diminishing its cost and giving high gauge of care [1]. Self-care - organization structure tries to send the restorative movement technique from specialist crashed into calm driven. Most by a wide margin of Europe countries give in financial issues conceivable and postponed empowering workplaces with a chose complete objective to help individuals United Nations office ability the tired impacts of DS [2]. Their territory unit a key perspectives expected to build keen home system as to such an extent as sanctioning individuals to fight with their cheery out-of-specialist's office mind. the dream of the arranged examine is to make up a system reading sight visible $\mid$ see able $\}$ of moveable stage from human administrations motivation behind view to permit patients extensively extra capacity to ID and tending to their conditions with respect to genetic blood dissipate. Amid this situation, the arranged system offers ongoing invigorating administrations edges and transference data for specialists patients coordinated effort that is relevant to the present patient's condition. DS could be a critical purpose behind concern and end of the day perseverance that influences tolerant welfare as a consequence of red thrombocyte (RBC) variety from the standard. Amid this particular situation, DS will impacts individuals through a social affair of familial issue because of the high outcome on the super molecule inside blood corpuscle that is named hemoprotein. For the principal a large portion of, the province of RBCs territory unit circle and flexible, that offer extra opportunity to movement through veins. In any case, RBCs with the general population United Nations organization encounters DS could show up as though edge device (C-shaped). In spite of the very certainty that DS can't be changed from individual to person, this illness non heritable to the tot by innate of edge apparatus hemoprotein either from every watchman or from one in all them evaluated as strange hemoprotein [3]. The disorder is caused by a genomic issue that decreases future by about forty five years amid a monstrous bit of made countries [4]. There region unit a fundamental upgrading in care and drugs, that have given a conceivable likelihood to gauge past forties or extra. In like manner, it's phenomenal for youngsters with DS to ask by recent years, especially in Africa and Asia principle lands [5]. DS can't be maintained a strategic distance from however with a true blue organization the disease are frequently well-kept. World Health Organization (WHO) expand that seven million arranged per fonce a year each year\} round the globe encounters either a uninheritable disease or from inherent quirk [6]. Extensively extra pushing could be im- 
mediate consequences of five-hitter of the world zone unit affected by hemoprotein issue, principally, DS and hypochromic pallor [7]. Reliable with National Health Services (NHS) examination information, there region unit 2.50 lakhs of publics with RBC sickness point of this investigation is proportional back costs and supply twenty four hours correspondence amongst specialists and patients. once the structure recognizes any imperative condition from the patient, it makes A modified message to the medicinal specialists that convey support with perfect decisions. In order to supply a quick reaction before situation winds up noticeably fundamental, the correspondence stage amongst patient and professional will be mechanically settled once persistent go surfing to the framework. Amid this case, the patient will be advised to require adjust amount of drug inside the reason for alleviating the separate of the ailment. Successful watch over DS needs standard perception utilizing a cell phone stage that would possibly affect relieving understanding ailment before continues to essential conditions. This paper gives and depicts a solid approach for distinguishing proof and perception of patients' journal to create higher nurture individuals who experience the ill effects of DS. Whatever is left of this paper is unionized as takes after. Area II can examine the current situation in medicinal parts though segment III demonstrates the arranged framework. Framework parts are represented in segment IV while the philosophy for characterizing DS patients from conventional patients is presented in area V. Segment VI shows the outcomes. At last, conclusion appears in segment VII.

\section{The Current Situation in Medical Sectors}

Existing work shows a spread of difficulties that is offered to the DS incorporated administration bundle. Barely any specialists have centered to disentangle this drawback with DS. Out-ofdoctor's facility mind is similarly a substitution space of research we'll focus on its application for DS administration framework. Consequently, it's fundamental to make framework which will bolster patients and specialists. As of late, the advancements in correspondence innovation are enhanced through exploitation sensible telephone application. the specific reason for correspondence between DS patients and specialists are regularly known in relationship with trading vital information and giving associated treatment. Concerning the correspondence innovation, tending experts have a tendency to talk with their patients to supply ideal therapeutic care and supply amend call upheld quick appraisal [9].Improved quiet specialist relationship approach means to stretch out adherence to guided treatment, construct confide in strategy, improve wellbeing results and nature of wellbeing. Cheng, et al [10] presented a DS administration framework for perception patient's condition through causing instant message in order to empower treatment adherence or gather self-evaluated medicinal data including to treatment changes. The perception framework depended on PDAs to contact patients wherever and whenever exploitation SMS messages. The impediment of their answer is its pertinence to grown-up patients exclusively. A portable journal stage for pre-adult in relationship with DS is arranged [11]. Their answer will give essential help for the utilization of electronic journal to follow side effects and agony for teenagers [11]. Their work bolstered using the net correspondence ability of dynamic cell phone upheld 3G/4G [12]. Past works demonstrated that there region unit a few confinements to deliver timeframe information with respect to DS ordinariness or variation from the norm [10], [11]. Regardless, this examination work was concentrating on of building extreme systems that is prepared to track infection and send minute data to clinicians by providing guided restorative care, particularly with pivotal conditions.

\section{Case System}

\section{1 (PTSD vs ASD)}

An accentuation was put on dissociative reactions happening at the season of the injury or presently in view of the injury survivors who confine their consciousness of the horrible accident and the subsequent feelings really block their capacity to process these feelings and consequently hinder normal recovery. 11 as such, the nearness of noteworthy dissociative side effects (talked about underneath) may anticipate a resulting determination of PTSD. Until the current time the improvement in information system and communication technologies and their applications in medical field had effectively changed the line of human lifestyle in relating with enhancing medical services and results .In this system it is crucial for patients who are effected by psychological disorders to be diagnosed in earlier stage .we are taking psychological disorder diseases such as ASD (Acute stress disorder), PTSD ( Post Traumatic Stress Disorder) .we consider these two psychological disorders these two diseases have many similar symptoms .In few days after the distressing event you may build up an apprehension issue called intense pressure issue. Intense pressure issue regularly happens inside couple of days of horrible event.it takes up to three days and can proceed up to one month. The reasons for occurrence of ASD are distress, observing, being confronted with one or more disturbing events can cause acute stress disorder. The events create panic, shock or powerlessness.it may likewise incorporate expiry, a danger of death to others, a risk of genuine mischief to others. ASD is dealt with by methods, for example, mental evaluation to decide your particular wants, hospitalization on the off chance that you are at risk of suicide, help with getting asylum, sustenance, and apparel ,mental training to show you about turmoil, introduction based treatments, hypnotherapy. Several persons with ASD are future spotted with PTSD. A diagnosis of PTSD is made if your symptoms continue for more than a month and cause a major amount of stress and difficulty functioning. PTSD manifestations may begin inside one month of an aggravating occasion, yet few times side effects may not see until years after the occasion. PTSD side effects are typically accumulated into four kinds: irritating got away, negative changes in considering and state of mind, and changes in physically and touchy responses. Indications can vary after some time or contrast from individual to individual. The manifestations may incorporate exasperating recollections, getting away, negative changes in considering and state of mind changes in physical and enthusiastic responses. Reasons for PTSD are unpleasant encounters, including the sum and seriousness of injury experienced out the lifetime. PTSD and ASD analysis requires an individual to encounter a horrific accident that involves together the goal and particular criteria talked about above (Criterion A1 and A2). Similar to PTSD, finding ASD requires no less than one side effect in reexperiencing the injury, for example, repeating contemplations, pictures, bad dreams, flashbacks, and serious passionate trouble upon introduction to indications of the injury that the individual showcases checked shirking of injury related musings or indications of the injury and that the individual presentations manifestations of tension or expanded excitement, for example, rest issues, peevishness, poor fixation, vigilance, and overstated startle . Similarly as with other DSM analyze, the aggravation must reason clinically essential torment or down to earth impedance and the unsettling impact isn't since of the impacts of a physiological matter or common helpful condition (Model G) and more awful spoken to by other disarray. The two highlights that partitioned the ASD assurance from PTSD are 1) the need for ASD that the particular involvement either in the midst of the shocking mishap or its result, no less than three of five dissociative signs (Model B;

desensitizing/separation, lessened consideration to one's environment, derealisation, depersonalization, and dissociative amnesia), and 2) the span run the show (Measure $\mathrm{H}$ ), which shows the unsettling impact ought to final no less than two days however final near to a month, and must happen interior a month of the damage. DSM-IV 
is unequivocal that either the manifestations must "cure inside a month after the finish of the awful mishap or the determination is changed." The comfort of ASD finding was most grounded for the cases in which (1) full ASD rules were met and (2) conditions where the person did not meet criteria for indeed subclinical ASD. Particularly, among people assembly full ASD criteria at the essential examination, 77.8 percent met full criteria for unending PTSD at the coming about appraisal and 22.2 percent did not meet criteria for indeed subclinical PTSD. Among people who did not meet criteria for indeed subclinical ASD at the covered up evaluation, 87.2 percent did not meet criteria for indeed subclinical PTSD at the coming about assessment and as it were 4.3 percent met full criteria for PTSD. The utility of the ASD finding was less beyond any doubt in events of subclinical ASD. Particularly, among people with subclinical ASD at the essential evaluation, 60 percent met full criteria for consistent PTSD.

\section{Methodology}

\subsection{Naïve Bayes:}

\section{Advantages}

It's comparatively easy to know and build

o It can be trained even with a tiny dataset

o It can be process very fast

o It's not sensitive to moot options

Disadvantages: Each feature is assumed as freelance, which isn't continuously the case

\section{Explanation:}

Naïve Bayes is one of the best choices for applications involving the classification test cases. We tried on working on several samples of Naive Bayes. While few were too sophisticated, some treated over Naive Bayes; however we have an example that we usually come across on the internet. It is capable of explaining the thought very well and runs through the easy maths behind it while not obtaining too technical.

Let us consider we have knowledge on one thousand psychological disorder patients. The disorders being PTSD, ASD or another disorder and we all know three symptoms of every disorder, whether it is depression, anger or insomnia as displayed within the table below:

Table 1: Psychiatric Disorder table

\begin{tabular}{|l|l|l|l|l|}
\hline Disorder & Depression & Anger & Insomnia & Total \\
\hline ASD & 400 & 350 & 450 & 500 \\
\hline PTSD & 0 & 150 & 300 & 300 \\
\hline Other & 100 & 150 & 50 & 200 \\
\hline Total & 500 & 650 & 800 & 1000 \\
\hline
\end{tabular}

Based on the Table 1 we can understand that;

Nearly, the outcome being an ASD is $50 \%$. Outcome being PTSD is $30 \%$ and outcome being any other disorder is $20 \%$. As per our coaching we can additionally address the following From 500 ASD patients $400(0.8)$ are depressed, $350(0.7)$ are having anger issues or $450(0.9)$ are insomniac. Out of 300 PTSD patients zero are depressed, a hundred and fifty $(0.5)$ are having anger issues and 300 (1) are insomniac. From the odd 200 other disorder patients, $100(0.5)$ are depressed, one hundred fifty (0.75) are having anger issues and $50(0.25)$ are insomniac.

1. This must be adequate enough to furnish evidence in accordance such that the new class introduced is classified accordingly.

2. Hence, let us address that we are devoted to the purposes of quantity concerned, yet we necessarily do it in accordance to predict the class. If we are instructed to classify based on depression, anger and insomnia, we perform an analysis using the components and subbing of the values for every outcome, to check whether it is an ASD, a PTSD or another disorder. The result provides the absolute best or likelihood (score or disorder) and the existence of the winner. Now since we've considered a basic example previously on Naive Bayes within action, you may without problem consult how many that execute keep utilized in accordance with Text Classification troubles such as like junk mail detection, emotion evaluation or categorization. You are able to examine greater in turn respecting the Text Classification into Text Analysis.

3. There you hold it, an easy clarification about Naive Bayes along an example. We appeal this helps.

\subsection{Machine Learning Algorithms:}

Supervised Learning: By what means the algorithm works?

These algorithms contains about a goal / result, variable or variable based which is in conformity with remaining predicted out of a fond - employ over predictors or independent variables. Using these sets regarding variables, we get a feature up to an expectation of map inputs in imitation of desired outcomes. The training method continues until the model achieves a desired degree over the education data. For our situation it is an arrangement method in light of Bayes 'hypothesis which is able considering and suspicion that indicators are autonomous of each other. For instance, a turmoil might be thought to be mental on the off chance that it includes indications that are identified with emotional wellness of a patient. Regardless of whether an element relies on another component of a similar set, a credulous Bayes classifier is equipped for considering these properties to autonomously add to the likelihood that the confusion is mental or not. Bayesian model is anything but difficult to fabricate and especially helpful in situations where there are substantial informational collections. In spite of the fact that having a basic model Naive Bayes is infamous in beating even few very modern demand approaches. The Bayes speculation gives us a strategy for finding out back probability $\mathrm{P}(\mathrm{C} \mid \mathrm{X})$ from $\mathrm{P}(\mathrm{C}), \mathrm{P}(\mathrm{X})$ and $\mathrm{P}(\mathrm{X} \mid \mathrm{C})$. Take a gander at the condition underneath (in 4.3).

\subsection{Equation Model:}

$P(C \mid X)=\frac{P(X \mid C) P(C)}{P(X)}$

Here,

$\cdot \mathrm{P}(\mathrm{C} \mid \mathrm{X})$ : back likelihood of course (target)

given indicator (property).

- $\mathrm{P}(\mathrm{C})$ : The earlier likelihood of lesson.

- $\mathrm{P}(\mathrm{X} \mid \mathrm{C})$ : is the probability which is the likelihood of indicator given course.

- $\mathrm{P}(\mathrm{X})$ is the earlier likelihood of predictor. There are certain steps/stages included in carrying out a Naïve Bayes Classifier.

Stage 1: Interpret the dataset to repeat table.

Stage 2: Construct Probability table by finding the probabilities like Cloudy probability and probability of target.

Stage 3: Utilize Naive Bayesian condition to examine the back probability for each lesson. The lesson with the most extraordinary back probability is the result of figure. Guileless Bayes utilizes a comparable method to anticipate probability of different course in light of distinctive characteristics. This calculation is for most portions utilized as a portion of substance characterization 
(more over message examination) and in issues having various classes for course of action purposes.

SYMPTOMS
Avoidance of people
Avoidance of people
Avoidence of people
Avoidence of people
Avoidence of people
Avoidence of people
Avoidence of people
Avoidence of people
Avoidence of people
Avoidence of people
Avoidence of people
Avoidence of people
Avoidence of people
Avoidence of people
Avoidence of people
Avoidence of people
Avoidance of people
Avoidance of people
Avoidance of people

TRAUMA
Death of known person or close person
witnessing a death
domestic abuse
domestic abuse
domestic abuse
domestic abuse
parental abandoment
divorce
divorce
divorce
prison stay
prison stay
prison stay
prison stay
prison stay
serious illness
serious illness
serious illness
terrorism

\section{Outputs}

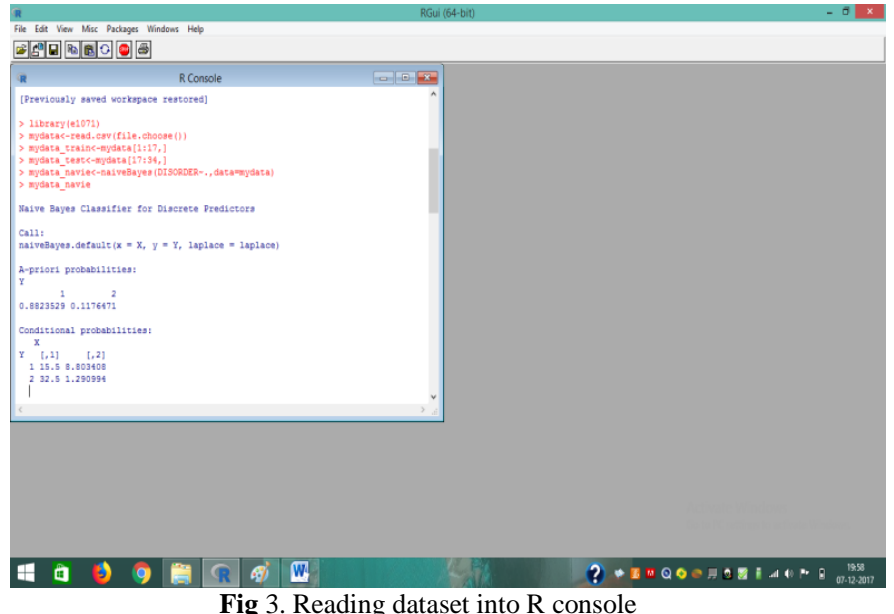

Fig 3. Reading dataset into R console

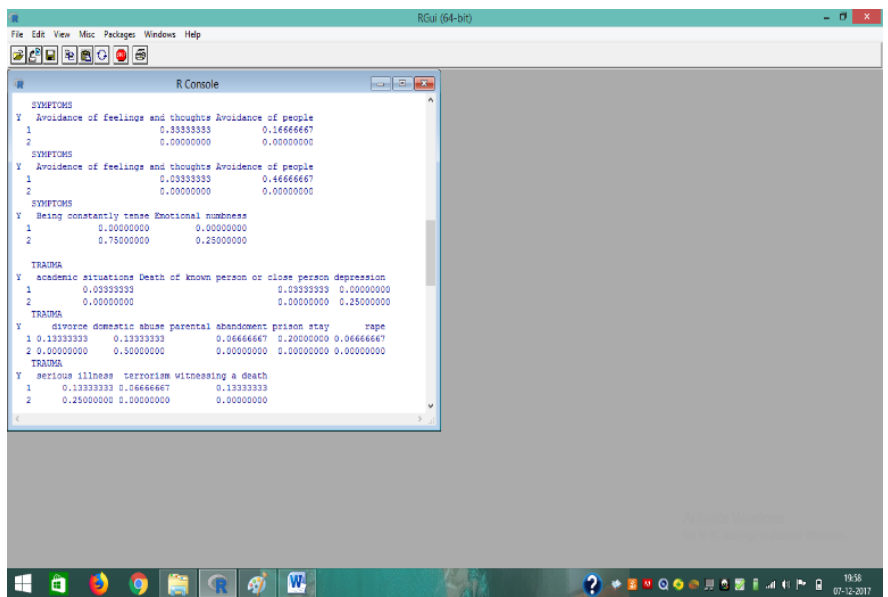

Fig 4. Output- Probability of each symptom leading to a psychiatric disorder

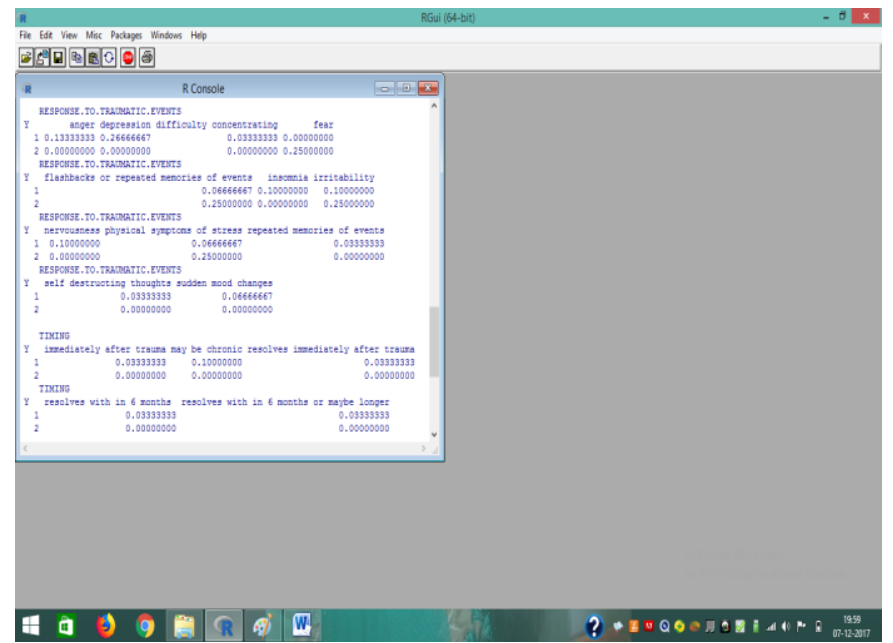

Fig 5. Output- Probability of response of traumatic events leading to a psychiatric disorder

\section{Results}

In this section, we have an opportunity to say about the outcomes that we have so far achieved in trying to create an assistance like system for psychiatrists, we used the $\mathrm{R}$ console to predict the probability whether the patient is having a particular disorder or not, based on the available dataset(Table 2). In the output screenshots, the 1 depicts the probability of the patient acquiring the Acute Stress disorder (ASD) and 2 depict the probability of the 
patient acquiring Post Traumatic Stress Disorder (PTSD). The dataset that we have used is regarding a patient's experience of a symptom to which there is a possibility that he can acquire a psychological disorder (Fig 3, 4 and 5).

\section{Conclusion}

So far our medical system has no means for predicting psychological disorders. We designed a system which is helpful to doctor to analyze the patient's status from which disorder he is suffering from based on his dataset which includes the types of disorders, trauma and responses to traumatic events. Further we can develop web based application using boosting algorithm for performance enhancement like logit boost algorithm. The web based application can be helpful in determining whether a patient is having a psychological disorder by taking symptoms, traumas and timings as inputs to classify the data into various disorders.

\section{References}

[1] J. Egea , M. González, and M. Menéndez, "EHealth Administrations Appropriation by European Common Professionals,"

in Surveying the Diverse Parts of Showcasing Hypothesis and Hone in the Jaws of Financial Vulnerability, H. E. Spotts, Ed., Springer Universal Distributing, 2015, pp. 205-210.

[2] A. Eleftheriou, M. Angastiniotis, D. Loukopoulos, C. Kattamis, and J. Meletis, "third Pan-European Conference on Haemo globinopathies and Uncommon Anaemias, 24-October 26 2012, LimassolCyprus," Thalassemia Reports, vol. 2, pp. 1-41, 2012.

[3] D.J. Weatherall, "The Part of the Acquired Clutters of Hemoglobin, the To begin with Molecular Diseases, in the Future of $\mathrm{Hu}$ man Hereditary qualities," Yearly review of genomics and human genetic qualities, vol. 14, pp. 1-24, 2013.

[4] I. Roberts and M. de Montalembert, "Sickle cell sickness as a worldview of development hematology: modern troubles for hematologists in Europe," Hematological, vol. 92, pp. 865-871, 2007.

[5] S. D. Grosse, I Odame, H.K. Atrash, D. D. Amendah, F.B.Piel, and T. N. Williams, "Sickle cell contamination in Africa: a expelled reason for early youth mortality," American journal of preventive medicate, vol. 41, pp. S398-S405, 2011.

[6] D.J.Weatherall,"The centrality of littler scale mapping the quality frequencies for the typical procured issue of hemoglobin," British journal of hematology, vol. 149, pp. 635-637, 2010.

[7] D. J. Weatherall, "The obtained diseases of hemoglobin are a rising around the world prosperity stack," Blood, vol. 115, pp. 43314336, 2010.

[8] J. de la Fuente and A. Mohammed, "Inescapability Of Nocurnal Enuresis and Proteinuria In Children With Sickle Cell Illness and Its Connection To Seriousness Of Excruciating Emergencies," Blood, vol. 122, pp. 4693-4693, 2013.

[9] C. Allayous, S. Clémençon, B. Diagne, R. Emilion, and T.Marianne, for "Machine Learning Calculations for Foreseeing Serious Emergencies of Sickle Cell Illness," 2008.

[10] A.V.Solanki, "Data Mining Methods Utilizing WEKA characterization for Sickle Cell Disease," IJCSIT) Worldwide Diary of Computer Science and Data Advances, vol. 5, pp. 5857-5860, 2014.

[11] A. A. Lazakidou, Web-based applications in therapeutic administrations and biomedicine vol. 7: Springer Science and Commerce Media, 2009.

[12] C.Feudtner, "Agreeable correspondence in pediatric palliative care an foundation for basic considering and fundamental administration ," Pediatric Clinics of North America, vol. 54, pp. 583-607, 2007.

[13] C.Cheng, R.C. Darker, L. L. Cohen, J. Venugopalan, T. H. Mixes, and M. D. Wang, "IACT An natural Wellbeing checking system to make strides psychotherapy for youthful individuals with sickle cell ailment," in Designing in Pharmaceutical and Science Society (EMBC), 35th Yearly Universal Conference of the IEEE, 2013, pp. $2279-2282$

[14] S. Yang, E. Jacob, and M. Gerla, "Electronic versatile e-Diary for youth with Sickle Cell Illness," in Shopper Communications and Organizing Conference (CCNC), 2012 IEEE, 2012. 